\section{More about the letter-frequency effect}

\author{
MILDRED MASON \\ Iowa State University, Ames, Iowa
}

Recently, this journal published a study by Appelman and Mayzner (1981) in which the authors examined previous letter-recognition studies to determine whether more frequent letters were easier to recognize than less frequent letters (the letter-frequency effect). They found a discrepancy between accuracy studies and reaction time (RT) studies, with only the latter providing evidence for a letter-frequency effect. From this, they concluded that letter familiarity has no effect on input coding, but may affect the comparison stage (and possibly the response stage).

As Appelman and Mayzner pointed out, although there are a large number of RT studies, the results are rarely provided for each letter separately. I would like to make such data available for the 12 uppercase consonant letters used in a series of recent RT studies (Mason, in press). Although letter frequency was not a variable of interest in these studies, the letter stimuli were selected on the basis of the Mayzner and Tresselt (1965) single-letter frequency counts in such a way that the results could be generalized over any possible letter-frequency variable. The letters TNSR represented high-frequency letters, CFMP represented intermediate-frequency letters, and JXQZ represented low-frequency letters.

The paradigm used was a letter-recognition RT task in which the subject saw a single letter (the target) for $2 \mathrm{sec}$. Following a delay of approximately $1 \mathrm{sec}$, another single letter (the display) appeared on the screen and the subject had to make a yes-no decision and indicate, by depressing one of two response keys, whether or not the display letter was the same as the target letter. The display remained on the screen until the response was made, and the dependent variable was RT measured from the onset of display to response. Table 1 presents the RT and error data (averaged over the 12 subjects who participated in the experiment) for the individual letters on positive trials as a function of letter frequency.

As can be seen from Table 1, there is no evidence of a letter-frequency effect either on RTs or errors. The correlation between letter frequency and RT was $r=$ -.25 , n.s., and the correlation between letter frequency and errors was $r=-.02$, n.s. At first glance, the failure to find an effect of letter frequency on RT might seem to be an anomaly. However, the RT studies surveyed by Appelman and Mayzner that did find letter-

The author's complete address is: Department of Psychology, Iowa State University, Ames, Iowa 50011.
Table 1

Mean RT (Milliseconds) and Error Rates (ER) for Individual Letters as a Function of Letter Frequency

\begin{tabular}{cccc}
\hline Letter & Frequency & RT & ER \\
\hline T & 8537 & 376 & .0333 \\
S & 5295 & 381 & .0167 \\
N & 5243 & 379 & .0500 \\
R & 5137 & 388 & .0833 \\
M & 2163 & 378 & .0500 \\
C & 2057 & 372 & .0500 \\
F & 1563 & 398 & .0500 \\
P & 1338 & 382 & .0167 \\
J & 131 & 387 & .0667 \\
X & 121 & 378 & .0833 \\
Q & 66 & 380 & \\
Z & 54 & 387 & .0333 \\
\hline
\end{tabular}

frequency effects on RT were primarily "samedifferent" experiments in which two letters were simultaneously presented. As Appelman and Mayzner point out, simultaneous "same-different" judgments need not entail letter recognition, since the two patterns can be compared without first being identified as particular letters. This was not the case in the present study, in which the first letter was held in memory and compared for a yes-no judgment with the second letter. In effect, the present study represents a successive "same-different" task, with a 1-sec interval between the two letters. The obtained RTs are a measure of the time needed to recognize the second letter, make the decision, and activate the response key.

In an admittedly ad hoc fashion, the present single-letter RT data support the Appelman and Mayzner conclusion that letter frequency does not influence input coding-more frequent letters are not recognized more rapidly than less frequent lettersbut rather that letter frequency may affect the comparison stage. What we have here are RT data that do not show a familiarity effect. The fact that they were obtained with a paradigm that makes comparison on a physical match level highly improbable would indeed suggest the comparison stage in the simultaneous task as the locus for RT differences attributable to letter frequency. The Appelman and Mayzner hypothesis that letter frequency affects the comparison stage is highly testable: RTs with the simultaneous "same-different" task should yield a letter-frequency effect, whereas RTs with the successive "same-different" task should not.

\section{REFERENCES}

Appelman, I. B., \& Mayznen, M. S. The letter-frequency effect and the generality of familiarity effects on perception. Perception \& Psychophysics, 1981, 30, 436-446.

Mason, M. Recognition time for letters and nonletters: Effects of 
serial position, array size, and processing order. Journal of Experimental Psychology: Human Perception and Performance, in press.

Mayzner, M. S., \& Tresselt, M. E. Tables of single-letter and digram frequency counts for various word-length and letter- position combinations. Psychonomic Monograph Supplements, $1965,1,13-32$.

(Manuscript received March 10, 1982; accepted for publication March 11, 1982.) 